\title{
探究计算机网络技术在电子信息工程中的应用
}

\author{
夏 星 \\ 中共淮安市委网信办，江苏 淮安 223000
}

[摘要]随着我国科技社会的不断进步发展，电子信息工程中的计算机网络技术水平也在不断提高。文章从计算机网络技术在 电子信息工程中的应用意义入手，探讨现代化电子信息工程发展路径，希望通过计算机网络技术的应用普及，提高电子信息 工程及其自动化工作效率, 为整个电子信息工程工作带来积极影响。

[关键词]计算机; 网络技术; 电子信息工程

DOI: $10.33142 /$ sca.v3i7.2682 中图分类号: E271 文献标识码：A

\section{Explore the Application of Computer Network Technology in Electronic Information Engineering}

\author{
XIA Xing
}

The Cyberspace Administration of CPC Huai'an Municipal Committee, Huai'an, Jiangsu, 223000, China

\begin{abstract}
With the continuous development of science and technology in China, the level of computer network technology in electronic information engineering is also improving. Starting from the application significance of computer network technology in electronic information engineering, this paper discusses the development path of modern electronic information engineering, hoping to improve the efficiency of electronic information engineering and its automation through the popularization of computer network technology, and bring positive influence to the whole electronic information engineering work.
\end{abstract}

Keywords: computer; network technology; electronic information engineering

\section{引言}

计算机网络技术是现代化高速发展的产物, 将其应用于电子信息工程, 是一个庞大的工程项目, 涉及的知识覆盖 面、管理环节、维修护理等相关问题, 都是极其复杂而又繁琐的。目前, 计算机网络技术已经成为电子信息工程项目 改革的重要方法, 成为人们生活中不可缺少的部分, 有效拓宽信息获取渠道, 在我国城市化建设中扮演着重要角色。

1 计算机网络技术在电子信息工程中的应用意义

计算机网络技术是指通过对数字化技术、电子信息技术、数据化技术等技术的整合，达到人工智能化技术进步的 目的。一般来说, 电子信息工程是一个庞大的工程项目, 其涉及的知识覆盖面、管理环节、数据更新、系统维护等相 关问题, 都是及其复杂而又繁琐的。计算机网络技术的应用, 能够实现电子信息工程自动化的标准统一, 可以通过建 立电子信息工程自动化模型等方式，加强对工程的控制管理。

只有加强计算机网络技术的管控, 重视相关系统的运行情况, 才能使整个电子信息工程长期处在正常工作状态中, 保证电子信息工程自动化工作的长远发展。打破传统模式下的发展禁锢, 体现了现代科学技术发展的重要意义, 实现 自动化技术的进一步升级创新。同时, 在一定程度上控制资源的利用效率, 减少资源的浪费, 降低人力资源和时间成 本的投入，实现企业收益最大化 ${ }^{[1]}$ 。

\section{2 计算机网络技术在电子信息工程中的应用探讨}

\section{1 完善网络防御系统}

目前, 网络病毒是对电子信息工程安全威胁最大的要素, 其通常具有较强的隐藏性, 在日常工作中难以被操作者 发现, 如图 3 所示。但在病毒发作时, 其对整个计算机系统的破坏性是巨大的, 如 “石头 2 病毒”、“DS. 3873 病毒”、 “Mai1-Bomb 病毒” 等。随着网络规模的不断扩大，在实现信息高速传递的同时，也为计算机病毒传播提供了条件。市 场上常见的杀毒软件能够消灭大部分影响较小的网络病毒, 但无法解决某些破坏性较强的病毒, 如前段时间出现的勒 索病毒, 通过对计算机系统的锁定进行勒索, 若拒绝进行转账就会造成计算机系统痽疾。这种病毒会严重地毁坏软硬 件系统, 严重影响了电子信息工程安全秩序, 挑战者网络法律的底线。

计算机网络网络技术的应用, 能够有效完善网络防御系统, 提高计算机系统安全防护等级。它能够实现用户在进 行网页浏览时的安全防护, 通过对网络系统安全监管, 实现对计算机病毒的有效拦截, 保障网络用户的信息使用安全。 具体体系构建时, 强调电子信息工程网络运行维护过程中对计算机系统起到保护作用, 实现电子信息工程的自动化防 
御, 即使没有操作人员进行指挥, 防护体系也能自主进行系统防护, 并且在操作人员进行人工防护时, 对相关监控、 信息、经验的情况进行智能化分析。将收集到的数据信息进行整合分析, 根据防护经验弥补电子信息系统漏洞, 并利 用合理合法的手段对网络信息安全的攻击者进行反击。

\section{2 自动网络技术设计}

计算机网络技术的应用, 能够帮助电子信息工程在日常运行过程中产生工作思维, 保证电子信息工程自动化控制 工作的顺利完成。自动网络控制取代了传统控制模式，大幅度提高控制力度，保证相关工作质量，完成电子信息工程 的高度自治化管理。在部分工作中实现了无人化运作, 提高工作效率的同时, 保证了工作的统一质量。在一些危险系 数大、操作难度高的工作中, 计算机网络技术可以规避环境影响, 直接对电子信息工程进行工作, 降低了人力资源的 必要性, 节约了生产成本。不仅如此, 在某些特定环境中, 计算机网络技术还能实现自我调节, 分析出各种问题相对 应的措施, 确保系统的安全性和科学性。自动网络技术设计还能实现工作资源的最优化配置, 不仅能在第一时间发现 相关设备的故障, 更能将系统的相关数据进行整理分析。简化日常办公流程, 加快管理办公效率, 降低人力资源和时 间成本的无效投入，有效地控制企业运行生产成本。

\section{3 重视分层系统设计}

在电子信息工程实际工作中, 因为其专业性较强, 对相关设计工作人员有着较高要求, 不仅需要掌握一定的计算 机理论, 还需要了解用多种不同系统之间的差异性。一般来说, 电子信息工程可以分为主系统与次系统两个部分, 利 用电计算机网络技术进行主次系统的自动化运行管理, 通过计算机网络技术中不同协议与接口之间的数据杂糅, 实现 电子信息工程的自动化控制。以某个电子信息工程为例, 其配置协议采用的为 AB 通讯协议, 且主系统配置为 Y-1ingk, 而次系统配置的是 ABMicroLogix1100PLC。利用电子信息技术中的多端口交流功能, 能够有效实现主系统与次系统之间 的数据传递, 且保障其全面性、真实性，从而实现对电子信息工程的作业实时控制 ${ }^{[2]}$ 。

电子信息工程系统设计较为繁琐, 内容结构较为复杂, 工作人员在进行设计工作时避免不了发生失误, 不利于后 续相关工作的开展。在实际工作中, 常利用计算机网络技术中的人工智能系统和遗传算法进行电子信息工程设计工作。 人工智能系统是通过一个或一组人工智能计算机程序, 在某些特定领域内, 应用大量的专家知识和推理公式, 来进行 复杂问题演算的现代化方法。它能根据项目作业的自身要求, 以及相关特性的需要, 完成最合理的设计。而遗传算法 是进化算法中的一种, 借鉴了进化生物学中的某些现象, 可以计算数学中用于解决最佳化的搜索算法。将其应用于电 子信息工程自动化工作中，改善了传统电子信息工作中先定标准再执行的复杂流程，极大程度上提高了作业效率。

\section{4 无线监控技术应用}

在计算机网络技术的支持下，电子信息工程能够在利用电子传感器进行相关数据传递的同时，进行数据识别、断 定、初步修改、决策等操作。对于电子信息工程作业环境中的网络安全、信息内容、传递效率等关键因素进行整合, 提高了摄像监控模块的监控功能与摄像监控模块的运用效应, 进而实现项目作业的自动化控制。此外, 还可以通过远 程监控系统进行实时控场, 在发生作业问题时, 利用计算机, 大幅度提升了电子信息工程的有效性及工作效率, 避免 了材料的损耗，降低了施工失误率。

例如, 可以通过远程摄像监控系统, 结合计算机网络技术在电子信息工程中的应用, 以及信息处理技术, 实时地 对电子信息工程运行状况进行监控。在系统运行出现异常情况的时候, 及时通过信息处理技术, 将发生的故障进行数 据整理, 并进行相关分析, 将分析结果由无线电传递给相关的检查人员。第一时间模拟出相关问题实验结果, 并提供 不同的解决方案，针对现行方案提出可行性整改措施。在事故发生的第一时间进行警报，缩短相关故障排查人员的检 查时间，提醒工作人员修理方向，提高电子信息工程工作效率，减少企业财产损失 ${ }^{[3]}$ 。

\section{3 结论}

作为目前科技领域范围中较为前沿的管理技术，基于计算机网络技术支持下的电子信息工程已逐渐融入人们的生 活, 发挥其高效化、智能化、科技化的技术特点, 为社会建设带来重大影响。强调计算机网络技术在电子信息工程中 的应用发展趋势研究，能够在强大的计算系统技术支持下，实现多项科研成果的融合发展，满足人们的不同应用需求。

\section{[参考文献]}

[1] 徐杰, 陆华才, 朱文明. 高素质应用型电气工程及其自动化专业人才培养模式改进的研究 [J]. 科技 风, $2020(14): 50-51$.

[2]赵畯成.浅谈电气工程及其自动化的智能化技术应用分析 [J].中外企业家, 2020 (06): 167 .

[3] 石元博, 魏海平, 黄越洋. 基于 PLC 的智能楼宇中电地热监控系统的设计与实现 $[\mathrm{J}]$. 工业仪表与自动化装 置, 2019 (03) : 45-48.

作者简介: 夏星 (1988. 8-), 男, 毕业院校: 常熟理工学院, 现就职单位: 中共淮安市委网信办。 